\title{
Indicación y resultados del tratamiento quirúrgico y rehabilitador en la ráfaga cubital reumática muy avanzada
}

\author{
G. Martínez Villén ${ }^{(1)}$, I. Herráiz Gastesi ${ }^{(2)}$, E. García y García ${ }^{(3)}$, \\ F. J. Manero Ruiz ${ }^{(4)}$, A. Herrera Rodríguez ${ }^{(5)}$ \\ Hospital Universitario Miguel Servet. Zaragoza \\ Servicio de Cirugía Ortopédica y TRaumatología (Unidad de Mano y Microcirugía) \\ SERVICIO DE REHABILITACIÓN Y REUMATOLOGÍA \\ (1) Profesor Asociado. Médico Adjunto. Unidad de Mano y Microcirugía del Departamento de C.O.T. \\ (2) MÉdico Residente deL DEPARTAMENTo de ReHabilitaCióN \\ (3) Jefe De Servicio del Departamento de Rehabilitación \\ (4) Jefe De Servicio del Departamento de Reumatología \\ (5) Catedrático. Jefe de Servicio del Departamento de C.O.T.
}

Correspondencia:

Dr. Gregorio Martínez Villén

Hospital Universitario Miguel Servet

$\mathrm{P}^{\mathrm{o}}$ Isabel la Católica 1-3

50009 Zaragoza

Teléfono 976765500

e-mail: gmarvil@terra.es

La ráfaga cubital de la mano es una deformidad que afecta a más del $36 \%$ de los pacientes con artritis reumatoide. Los motivos para la indicación quirúrgica son controvertidos. Se han descrito numerosos procedimientos para la reconstrucción de partes blandas y para la artroplastia de las metacarpofalángicas. Siguiendo el orden de preferencia establecido en la artritis reumatoide de restaurar la función, aliviar el dolor y corregir la deformidad, fueron intervenidas quince manos con una ráfaga cubital avanzada mediante centrado tendinoso y artroplastia protésica o artrodesis de las metacarpofalángicas. Después de la cirugía se aplicó un protocolo intensivo y específico de rehabilitación. La petición de los pacientes en relación a la cirugía fue la recuperación funcional y la mejora cosmética; el dolor no constituyó una preferencia, y cuando estuvo presente fue sustancialmente aliviado o desapareció.

Palabras clave: mano reumática, ráfaga cubital, cirugía, rehabilitación.
The ulnar drift is a frequent hand deformity that affects more than $36 \%$ of the patients with rheumatoid arthritis. Surgical indications are controversial, and several procedures for soft tissue reconstruction and metacarpophalangeal joint arthroplasty have been described. According to criteria for surgery in restore function, relieve pain and correct deformity in rheumatoid arthritis, fifteen hands with advanced ulnar drift were operated for tendon-rebalancing, prosthetic metacarpophalangeal joint arthroplasty or arthrodesis. Intensive and specific postoperative rehabilitation was programmed. In our cases, after functional gain, the request of the patients regarding surgical treatment was hand appearance. The pain was not a preference, and when it was present, was outstandingly relieved or it disappeared during the postoperative period.

Key words: rheumatoid hand, ulnar drift, surgery, rehabilitation. 


\section{INTRODUCCIÓN}

Aproximadamente dos millones de personas padecen artritis reumatoide (AR) en los países de la Comunidad Económica Europea. En el $70 \%$ u $80 \%$ de los afectados, la mano es lugar preferente de manifestación de la enfermedad, que se caracteriza por la aparición de deformidades. La desviación cubital de los dedos largos - también conocida como ráfaga cubital - , es la más frecuente, y está presente entre un $36,5 \%^{1} \mathrm{y}$ un $50 \%^{2}$ de las ocasiones.

El inicio de la deformidad es una sinovitis metacarpofalángica (MCF), primero proliferativa y después destructiva. El proceso inflamatorio cronificado dará paso a alteraciones en los elementos de contención articular, que se hacen laxos y se rompen; al debilitamiento de la expansión de los Mm interossei que mantiene los tendones extensores centrados sobre las MCF, y a la usura de la superficie condral, que se ulcera y fragmenta. A grandes rasgos, el resultado final es la luxación o subluxación palmar de la primera falange (F1); la luxación de los tendones extensores en la vertiente cubital de las MCF; la retracción de los intrínsecos cubitales; la retracción de la expansión cubital de los Mm interossei y la relajación de la expansión radial de los mismos.

Todas estas modificaciones se agrupan en tres grados según la clasificación de Allieu ${ }^{3}$ : I) sinovitis y desviación cubital sólo en flexión MCF; II) desviación cubital avanzada en extensión y en flexión con subluxación anterior de la F1, y pocas o ninguna lesión osteoarticular perceptible radiológicamente y, III) desviación cubital avanzada, parcialmente fijada con destrucción articular.

Dado que el tratamiento quirúrgico en la AR aporta una solución paliativa en el contexto de una enfermedad progresiva, sus indicaciones y utilidad han sido desde hace mucho tiempo motivo de controversia entre cirujanos de mano y reumatólogos, quienes se han achacado un desconocimiento mutuo de las alternativas que ofrecen ambas especialidades ${ }^{4}$. En la actualidad, el procedimiento quirúrgico es ampliamente aceptado para las alteraciones presentes en los grados II y III de Allieu ${ }^{3}$, con argumentos discutibles en lo que se refiere a la sinovectomía 


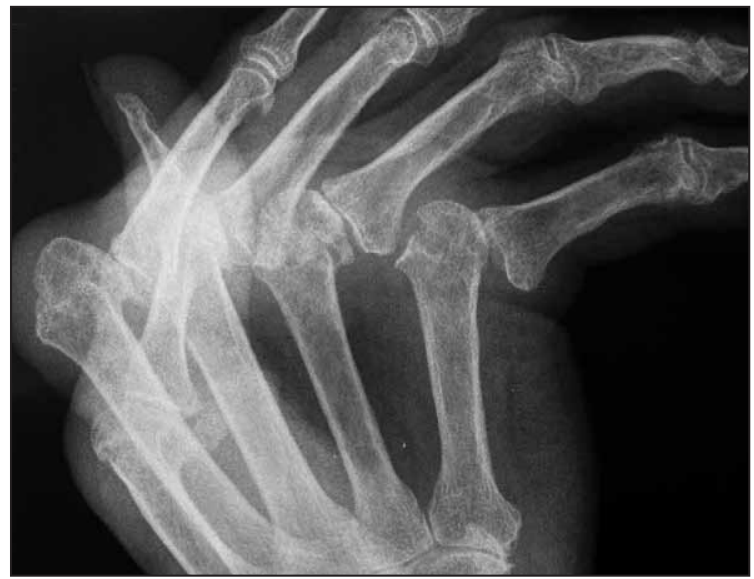

Figura 1. Proyección radiológica oblicua de las MCF en una ráfaga de grado III de la clasificación de Allieu ${ }^{3}$, con deterioro osteocondral de grado III a V de la clasificación de Larsen-Dale-Eek ${ }^{6}$. Obsérvese la subluxación palmar acentuada de la primera falange de los dedos.

subjetivamente por la dificultad para realizar actividades manuales de la vida cotidiana o profesional, y objetivamente mediante goniometría y dinamometría.

3. Presencia de dolor, cuya intensidad fue estimada con la Escala Visual Analógica (EVA).

4. Alteración estética.

Basados en los criterios mencionados, la indicación quirúrgica se hizo:

1. En casos con una ráfaga de tipo II y III de Allieu ${ }^{3}$, observando cinco manos con un grado II y 10 manos con un grado III. Estas manos arrojaron un total de $60 \mathrm{MCF}$ de los dedos largos implicadas en la deformidad: 20 con escaso o ningún deterioro osteocondral en la clasificación de Larsen-Dale-Eek ${ }^{6}$, y 40 con lesión de grado III (anormalidad destructiva media), a V (anormalidad mutilante) (Figura 1), lo que constituyó el argumento para la colocación de prótesis en $36 \mathrm{MCF}$, y la realización de artrodesis en otras cuatro.

2. En casos con limitación tanto para el agarre de objetos esféricos o cilíndricos (como un vaso), como para la ejecución de una pinza «suficiente» entre el pulgar y los dedos largos (abrir una puerta con llave, abrocharse los botones o escribir), $\mathrm{u}$ otros gestos manuales habituales (abrir un tarro o coger un plato). Estos aspectos fueron constatados en todos los pacientes, tres de los cuales alcanzaban el grado de invalidez.

3. La alteración estética fue también un factor coadyuvante que condujo a la determinación

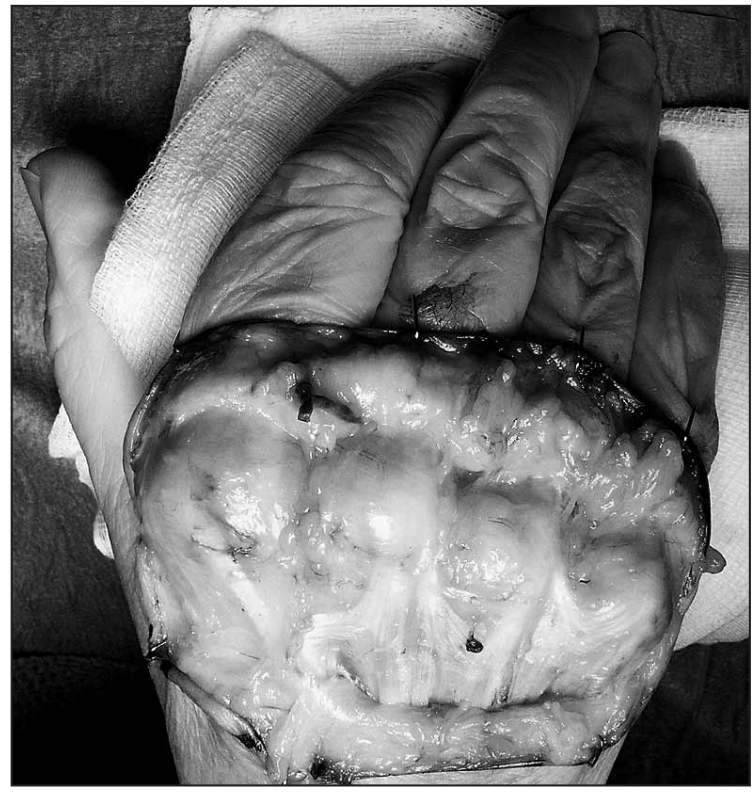

Figura 2. Imagen intraoperatoria en la que se puede percibir la luxación de los tendones extensores en la vertiente cubital de las $\mathrm{MCF}$.

del tratamiento quirúrgico, tanto de manera objetiva como subjetiva. En este sentido, todos los enfermos coincidieron en hacer una valoración negativa sobre el aspecto de sus manos. Cuatro de ellos manifestaron, incluso, repercusión psicológica.

4. La presencia de dolor no fue un factor determinante para la indicación quirúrgica en todos los pacientes. Así, en cuatro casos con un valor preoperatorio medio en la EVA de 5,7 $(\mathrm{r}=5-6)$, la intensidad del dolor se estimó como motivo suficiente para la intervención, mientras en siete no, con un valor preoperatorio medio en la EVA de 1,6 $(r=0-3)$.

En los nueve casos en los que se descartó la intervención: cinco presentaban una ráfaga en grado I de la clasificación de Allieu ${ }^{3}$, con buena respuesta al tratamiento médico; tres presentaban una ráfaga en grado II-III, con indicación quirúrgica rechazada por el enfermo, y uno presentaba una ráfaga en grado III, cuya intervención fue pospuesta para dar prioridad a una artroplastia protésica de cadera.

En las 15 manos de los 11 paciente intervenidos quirúrgicamente se consiguió la corrección quirúrgica de la desviación cubital y de la luxación o subluxación palmar de la F1, así como de la luxación del sistema extensor (Figura 2), mediante: 1) tenotomía de los intrínsecos del lado 


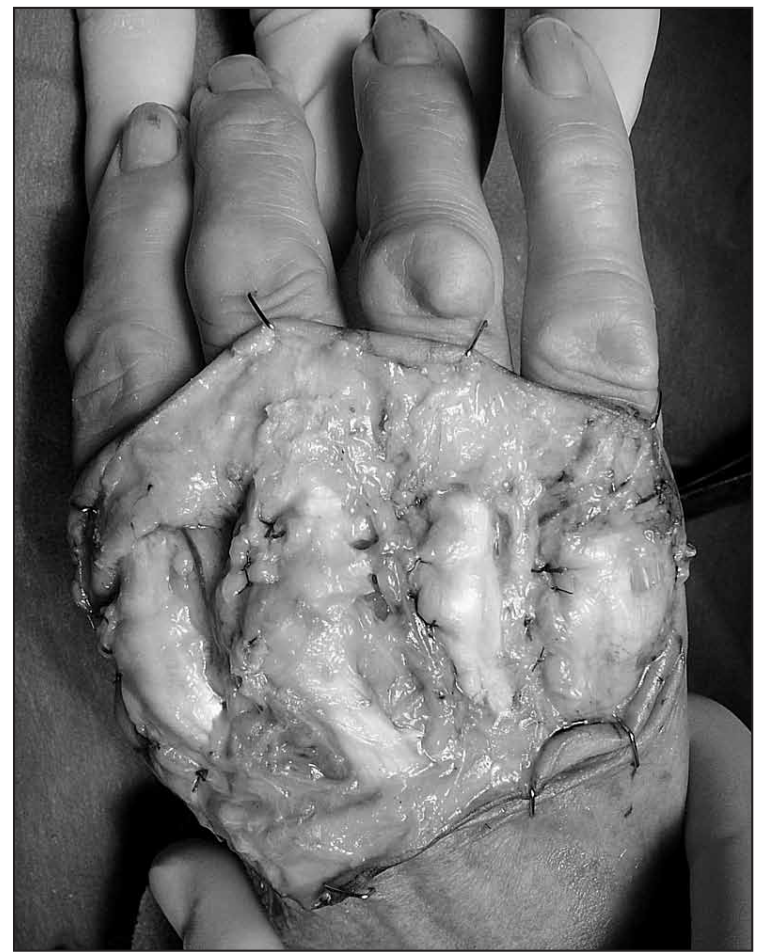

Figura 3. Centrado de los tendones extensores tras las incisiones de relajación en la expansión del lado cubital de los interossei y la plicatura de los mismos en el lado radial, en una mujer con ráfaga cubital de grado III (mismo caso de la figura 7).

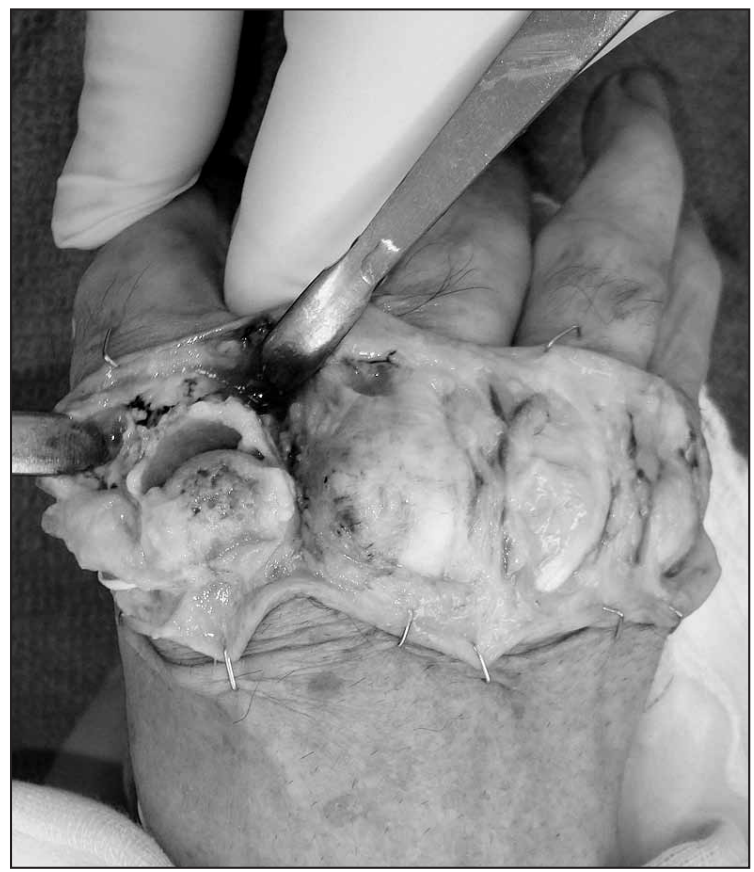

Figura 4. Lesión osteocondral de la MCF2 con exposición de hueso subcondral en un varón con ráfaga cubital de grado III. Obsérvese bien definida la luxación asociada de los tendones extensores en la vertiente cubital de las MCF 3 y 4 . cubital y del abductor digiti minimi, 2) artrolisis en el complejo capsular volar y, 3) incisiones de relajación en la expansión del lado cubital de los interossei con plicatura en el lado radial (Figura 3).

En las articulaciones con intensa lesión osteocondral MCF (Figura 4), las prótesis colocadas fueron de un elastómero de silicona Siflex II con el centro de rotación en la bisagra desplazado hacia palmar (Sistema Avanta Orthopaedics ${ }^{\circledR}$ ) (Figura 5). Si por el contrario se optó por la artrodesis MCF, ésta se llevó a cabo con agujas de Kirschner cruzadas, constatando antes una función satisfactoria de las interfalángicas proximal (IFP) y distal (IFD). Cuando no hubo lesión osteocondral asociada a la deformidad, o fue mínima, se actuó únicamente sobre partes blandas para lograr un centrado de los tendones extensores y una alineación adecuada del eje digital. La ráfaga cubital estuvo asociada en siete manos a una deformidad en ojal o «boutonnière» (Nalebuff tipo I) de la columna del pulgar, que se corrigió en el mismo tiempo operatorio. La sinovectomía se practicó según necesidad. To-

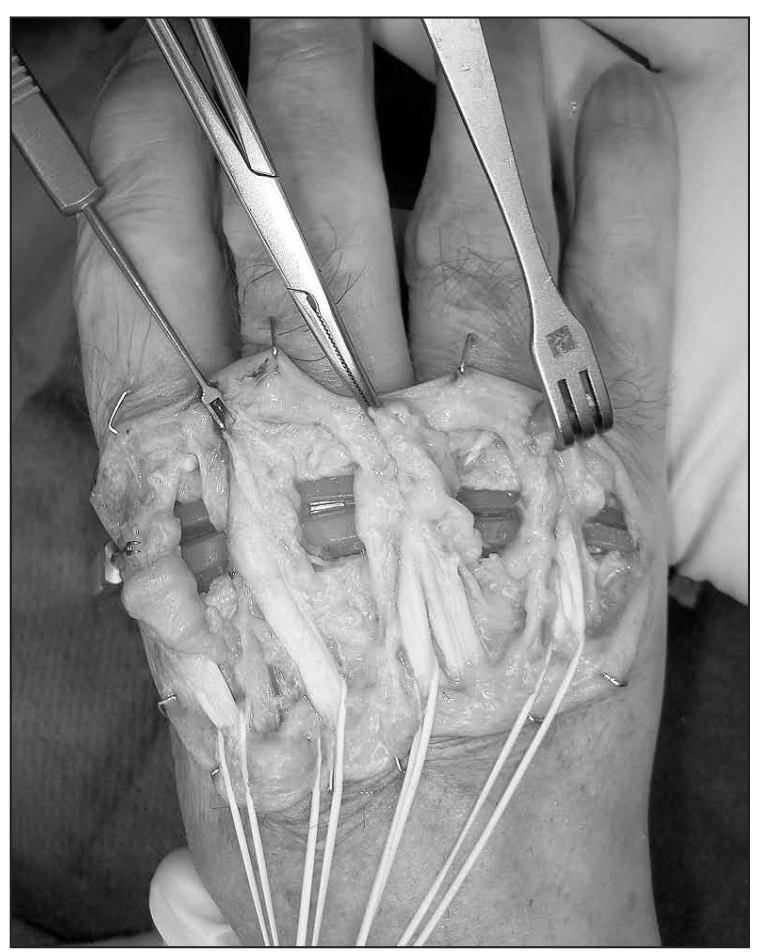

Figura 5. Mismo caso clínico de la figura 4 después de la colocación de cuatro prótesis de un elastómero de silicona en las MCF de todos los dedos largos de la mano derecha. 


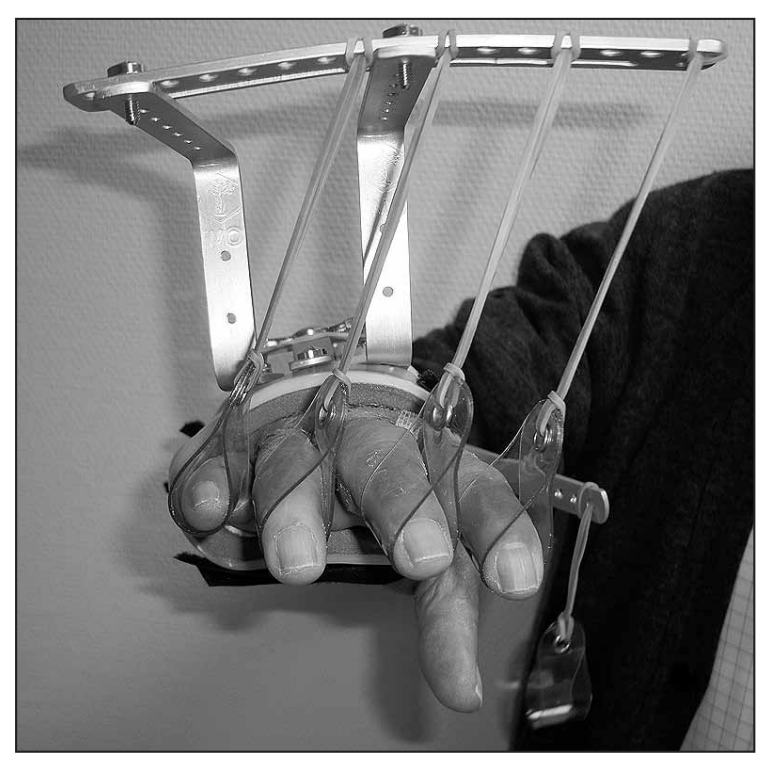

Figura 6. Ortesis dinámica para la flexión activa y extensión pasiva, mostrando sus hamacas digitales traccionadas por elásticos en sentido radial durante el postoperatorio de una ráfaga cubital de grado III.

dos los enfermos fueron operados por el mismo cirujano (GMV).

Concluida la cirugía, en las articulaciones no artrodesadas se colocó una férula de yeso antebraquio-palmar durante dos semanas, con las MCF en extensión evitando la hiperextensión, y la F1 en discreta desviación radial, quedando las articulaciones IFP e IFD libres para su movilización precoz. Retirada la sutura, esta férula fue sustituida por una ortesis dinámica dorsal tipo Mannerfelt que acababa en las MCF y dejaba la muñeca en posición funcional. Las hamacas digitales mantenían pasivamente la extensión completa de las MCF gracias a la tracción con elásticos desde las F1 en sentido discretamente radial (Figura 6). La flexión fue activa contra la resistencia de dicho elástico, progresando desde algunos grados en los primeros días del ferulado dinámico, hasta el definitivo rango de flexión al final de la RHB con este aparato. El calibrado de la resistencia se consiguió alargando, acortando o modificando el grosor del elástico. La ortesis dinámica se utilizó seis semanas durante el día, dejando de ser aplicada progresivamente durante las dos últimas semanas de este periodo. La movilización pasiva de los dedos sin el ferulado dinámico, la realizó el propio cirujano o el médico rehabilitador a partir de las tres semanas de la in- tervención quirúrgica. Para el descanso nocturno y a lo largo de los 4,5 a 7,5 meses posteriores a la cirugía, el paciente utilizó una ortesis estática que mantenía la extensión completa tanto de las MCF como de las IF.

Las MCF artrodesadas se mantuvieron bloqueadas 6 semanas con las agujas de Kirschner, permitiéndose la movilización activa y pasiva de las interfalángicas desde el postoperatorio inmediato. Dado que la artrodesis suprime la movilidad, estas articulaciones no han sido consideradas en la valoración funcional MCF postoperatoria, pero si en la corrección del defecto estético. El tiempo de seguimiento medio después de la cirugía fue de 24 meses $(r=45-8)$.

\section{RESULTADOS}

La imagen radiológica preoperatoria de subluxación o luxación de la F1 (Figura 1), e intraoperatoria de luxación de los tendones extensores en el lado cubital (Figuras 2 y 3) fue constante, corrigiéndose en todos los dedos tras la cirugía. El ángulo medio de la ráfaga cubital pasó de una media de $40^{\circ}$ en el preoperatorio a $18^{\circ}$ en el postoperatorio (Figura 7).

Desde un punto de vista biomecánico, la rectificación del eje digital se tradujo en una destacada mejora funcional, con una importante corrección estética objetiva y subjetiva. En las $36 \mathrm{MCF}$ en las que se centraron los tendones extensores y se colocaron endoprótesis, el déficit medio de extensión activa fue de $19^{\circ}$, siendo $58^{\circ}$ la media alcanzada para la flexión. En las $20 \mathrm{MCF}$ en las que sólo se actuó sobre partes blandas, el déficit medio de extensión activa fue de $15^{\circ}$, siendo $65^{\circ}$ la flexión media alcanzada. La fuerza media para la presa fue de $10 \mathrm{Kg}, \mathrm{y}$ no experimentó prácticamente modificaciones en relación al preoperatorio. La alineación conseguida en las cuatro MCF mediante artrodesis en posición fisiológica fue completa, quedando el radio digito-metacarpiano estable.

El dolor se alivió o desapareció en el postoperatorio en aquellos pacientes que lo habían manifestado antes de la cirugía, pasando de un valor en la EVA de 5,7 a otro de 0,17.

Según la impresión del enfermo, en cinco manos se obtuvo un resultado muy satisfactorio, 

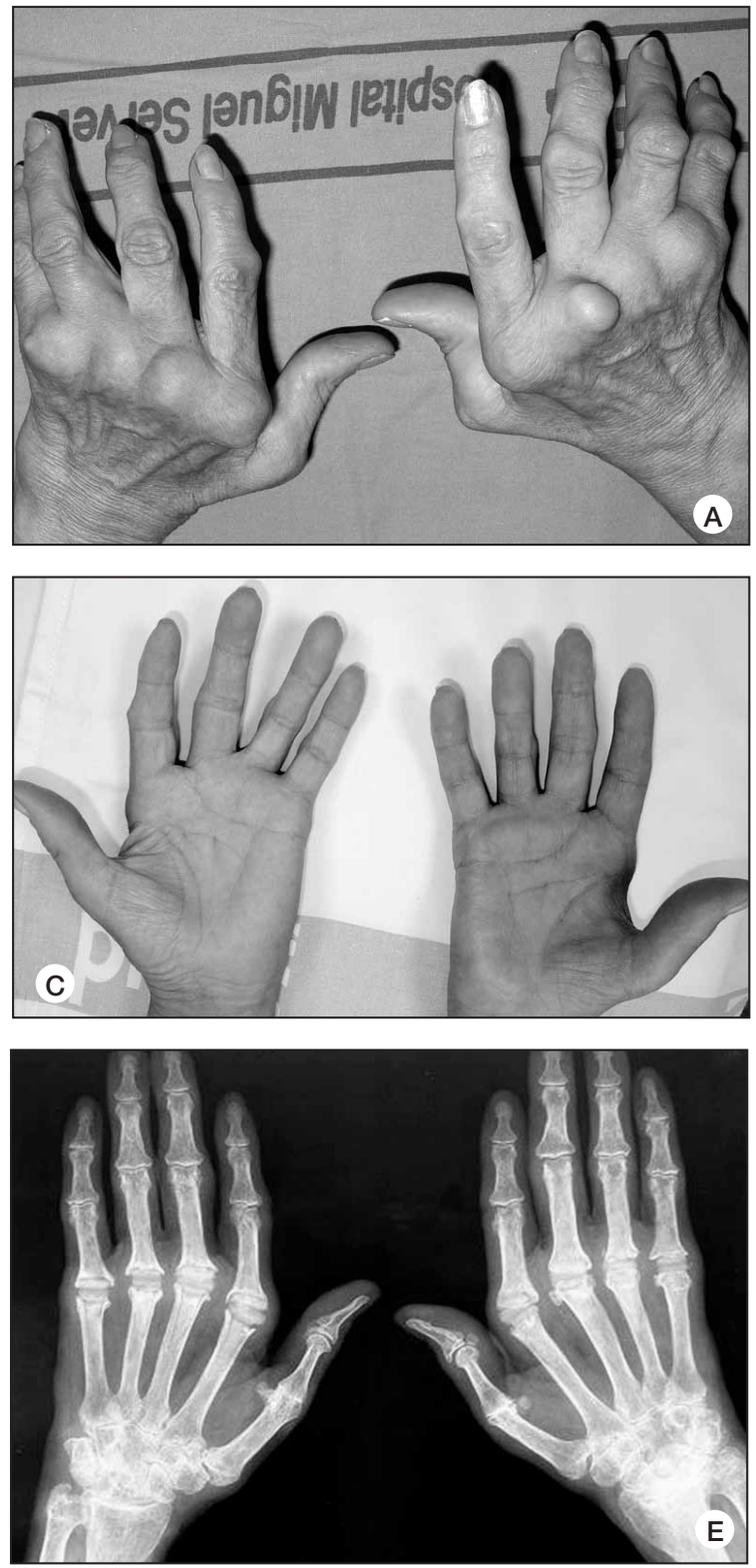

satisfactorio en siete, poco satisfactorio en dos e insatisfactorio en una. Los motivos de la satisfacción se basaron en el resultado funcional y estético. Los motivos de la insatisfacción fueron la recidiva de un flexus en siete MCF de dos manos en las que sólo se centraron los extensores, y un defecto de extensión superior a la media en otra mano con deformidad extrema.

\section{DISCUSIÓN}

En los últimos 10 años el desarrollo de agentes farmacológicos como el Etanercept, el In-
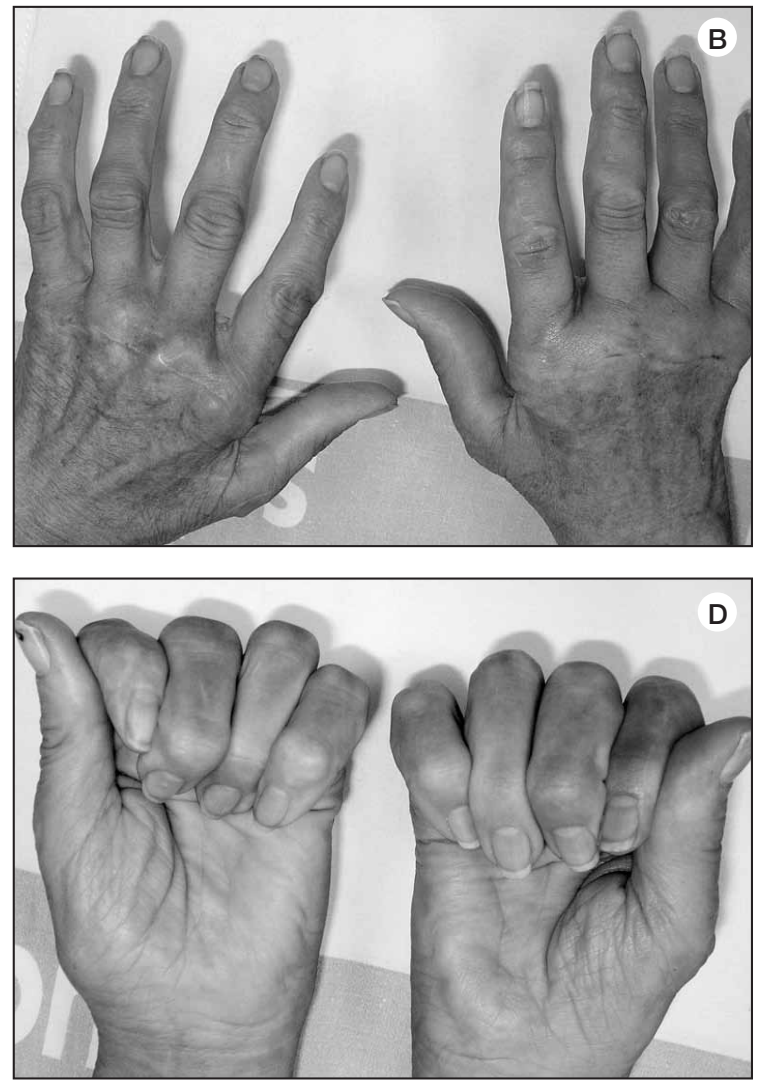

Figura 7. Caso clínico: (A) Grado III de una ráfaga cubital bilateral en el estado preoperatorio. (B) Corrección dorsal, $(C)$ palmar, $(D)$ rango de flexión y $(E)$ aspecto radiológico a los 12 meses de la cirugía en la mano derecha y a los 19 meses en la mano izquierda. En ambos pulgares también fue corregida la deformidad en ojal (boutonière).

fliximab o el Adalumimab, han favorecido notablemente el tratamiento de la AR. Gracias a ello, se ha conseguido controlar mucho mejor la sinovitis, siendo menor el número de enfermos jóvenes que precisan cirugía ${ }^{7}$. Este progreso no ha servido para conciliar la visión discrepante de cirujanos y reumatólogos sobre la indicación quirúrgica en la mano reumática ${ }^{4}$. Este desacuerdo hace que muchos pacientes de edad media o avanzada, a menudo lleguen a la consulta quirúrgica con las manos en un estado de deformidad grave o incluso extrema. En esta situación:

1. La capacidad de adaptación está sobrepasada y hemos encontrado algunos casos de verdadera invalidez y afectación psicológica.

2. La medicación ya no puede prevenir la deformidad - plenamente establecida - , ni corregirla, ni devolver la función. 
3. Las ortesis sólo sirven para aliviar el dolor cuando existe.

4. la RHB es útil para mantener un determinado rango de movilidad, pero con frecuencia también es inoperante al encontrase la articulación fijada o rígida.

Son manos en las que la única alternativa terapéutica, desde nuestro punto de vista, es quirúrgica.

Tradicionalmente, los pilares en los que se sustenta la indicación quirúrgica para corregir la ráfaga cubital son, en este orden: la restauración de la función, el alivio del dolor y la corrección de la deformidad ${ }^{8,9}$. En nuestros pacientes, el hecho de tener una ráfaga avanzada según la clasificación de Allieu ${ }^{3}$ (grados II y III), una disfunción mecánica acentuada, y una alteración estética acusada, han sido las razones fundamentales que han conducido al cirujano y al enfermo a elegir la opción quirúrgica. El dolor, siendo un factor significativo en algunos casos, no ha ocupado el lugar preponderante que habitualmente se le concede. Creemos que la explicación se halla en que la mayor parte de los pacientes intervenidos quirúrgicamente por nosotros, estaban en una fase de la AR denominada «quemada» («burnt out» de los anglosajones), crónica inactiva, o de colapso esquelético según Steinbrocker ${ }^{10}$. En esta fase, el dolor tiende a disminuir o incluso desaparecer, al contrario de lo que sucede en los estadios proliferativo y destructivo precoz de la sinovitis $^{10,11}$. Independientemente del momento evolutivo, la sintomatología dolorosa suele estar enmascarada por el uso habitual de fármacos, que se seguirán administrando como tratamiento de fondo controlado por el reumatólogo.

La cirugía correctora de la desviación cubital en las MCF, asociada a artroplastia protésica, logra un rendimiento articular satisfactorio con las técnicas que hemos utilizado. De esta forma hemos conseguido rangos de movilidad que en términos medios no difieren de otros trabajos publicados utilizando los mismos o similares elastómeros de silicona ${ }^{1,2,4,8,1213}$. Esto ha permitido una función en general mejorada en relación a la situación preoperatoria, si exceptuamos la potencia para la presa, que se ha mantenido invariable. Este último dato adquiere un valor secundario por la escasa exigencia funcional de estos enfermos, que aprecian más una movilidad útil para las labores fundamentales de la vida cotidiana, que un ejercicio de fuerza inalcanzable. Llama la atención en nuestros resultados, que la ganancia de movilidad fue algo mayor en aquellas articulaciones en las que no se colocaron endoprótesis y sólo se centraron los tendones extensores; sin embargo, la recidiva del flexus MCF como principal motivo de insatisfacción, también ha tenido lugar con este tipo de intervención. Este hecho nos hace reflexionar sobre la indicación de efectuar un simple centrado del sistema extensor cuando no coexiste lesión osteocondral asociada.

En la recuperación de la función postoperatoria, la RHB ha sido prioritaria para consolidar los mecanismos quirúrgicos correctores, obtener el máximo rendimiento funcional y educar al paciente en el contexto de su plurideficiencia. Este postulado obvio, llega a convertirse a veces en un obstáculo difícilmente salvable por la presión asistencial en los servicios de RHB de los grandes centros hospitalarios de referencia. No es raro, por tanto, que a veces sea el mismo cirujano quien conduce esta fase del tratamiento postoperatorio.

El protocolo de RHB seguido por nosotros no discrepa de las líneas generales de otros planteamientos ${ }^{12-15}$, con algunas observaciones. Así, consideramos deseable la movilización activa precoz IFP-IFD para mantener el deslizamiento tendinoso y aliviar el edema postoperatorio, pero preferimos retrasar el ferulado dinámico de las MCF hasta la retirada de la sutura a los 14 días de la intervención por término medio. Con esta actitud evitamos el apoyo mal tolerado de los aparatos cerca o sobre la herida quirúrgica, que no favorece la cicatrización del delicado tejido reumático, ni dispensa la comodidad necesaria en el postoperatorio inmediato. Consideramos que prolongar la inmovilización MCF hasta la retirada de la sutura, en la mano reumática no aumenta el riesgo de rigidez articular, ya que la neocápsula fibrosa creada en torno al elastómero de silicona, no ha tenido tiempo ni de formarse ni de quedar en una retracción que limite más adelante la flexión. Se acepta que el implante está definitivamente encapsulado a las seis semanas, momento en el que la aplicación diaria de la férula dinámica empieza a ser discontinua. La movilización pasiva no ferulada nos parece 
inoportuna hasta las tres semanas de la intervención, cuando el implante es más estable y su luxación menos probable. En lo que concierne a las ortesis estáticas para el reposo nocturno, somos partidarios de aplicarlas tanto tiempo como sea posible, especialmente si se retrasa la consecución de un grado de extensión MCF adecuado, o si se observa algún signo de recurrencia de la desviación cubital.

Respecto a la alteración estética de la deformidad, siempre se ha defendido su menor trascendencia de cara a la indicación quirúrgica ${ }^{8}$. Es obvio, no obstante, que la corrección de la desviación para ganar función, lleva implícita una mejora cosmética incuestionable. A pesar de todo, un reciente trabajo de Mandl et al. ${ }^{16}$, encuentra que el aspecto externo de la mano es el factor mejor relacionado con el grado de satisfacción del paciente tras la artroplastia MCF en la AR. Estos mismos autores manifiestan que el deseo subyacente de mejora estética, puede ser la primera razón por la que el enfermo solicita la cirugía. Sin coincidir completamente con este planteamiento, asumimos que la ganancia funcional ha de ser el objetivo principal tanto del cirujano como del paciente, pero nos ha llamado la atención en nuestros casos, que la petición de modificar favorablemente la imagen de sus manos, ha sido el segundo motor de la intervención.

\section{BIBLIOGRAFÍA}

1. Moutet F, Corcella D, Pradel P, et al. Surgery of the MCP joints in rheumatoid arthritis. En: Trail I, Hayton M (Ed). Surgery of the rheumatoid hand and wrist. Amsterdam: Elsevier BV, 2006: 118-28.

2. Sollerman C. How I do it-MP joint arthroplasty. En: Trail I, Hayton M (Ed). Surgery of the rheumatoid hand and wrist. Amsterdam: Elsevier BV, 2006: 144-53.

3. Allieu Y, Brahin B. Las deformidades de la mano reumatoide y sus tratamientos. Documenta Geigy (Ed). Barcelona: Viking S A, 1988.

4. Alderman AK, Chung KC, Kim $\mathrm{HM}$, et al. Effectiveness of rheumatoid hand surgery: contrasting perceptions of hand surgeons and rheumatologists. J Hand Surg Am, 2003; 28: 3-11.

5. Brewerton DA. Hand deformities in rheumatoid disease. Ann Rheum Dis, 1957; 16: 183-97.

6. Larsen A, Dale K, Eek M. Radiographic evaluation of rheumatoid arthritis and related conditions by standard reference films. Acta Radiol Diagnosis, 1977; 18: 481-91.
7. Sturrock RD. Update on the pathogenesis of rheumatoid arthritis. En: Trail I, Hayton M (Eds). Surgery of the rheumatoid hand and wrist. Amsterdam: Elsevier BV, 2006: 1-8.

8. Chung KC, Kotsis SV, Kim HM, et al. Reasons why rheumatoid arthritis patients seek surgical treatment for hand deformities. J Hand Surg Am, 2006; 31: 289-94.

9. Gwilliam L. Outcome measures following surgery to the rheumatoid hand. En: Trail I, Hayton M (Eds). Surgery of the rheumatoid hand and wrist. Amsterdam: Elsevier BV, 2006: 43-55.

10. Biese J. Therapist's evaluation and conservative management of rheumatoid arthritis in the hand and wrist. En: Mackin EJ, Callahan AD, Skirven TM, et al. (Eds). Rehabilitation of the hand and upper extremity. $5^{\mathrm{a}}$ ed. St. Luis: Mosby, 2002: 1569-82.

11. Smith P. Lister's The Hand. $4^{a}$ ed. London: Churchill Livingstone, 2002: 331-97.

12. Manuel JLM, Weis APC. Silicone metacarpophalangeal joint arthroplasty. En: Strickland JW y Graham TJ (Eds). The Hand, 2nd ed. Philadelphia: Lippincott Williams \& Wilkins, 2005: 391-403.

13. Trail IA. Silastic metacarpophalangeal joint arthroplasty. En: Trail I, Hayton M (Eds). Surgery of the rheumatoid hand and wrist. Amsterdam: Elsevier BV, 2006: 129-43.

14. Feldon P, Terrono AL, Nalebuff $E A$, et al. Artritis reumatoide y otras enfermedades del tejido conjuntivo. En: Green DP, Hotchkiss RN, Pederson WC, et al. (Eds). Green's Cirugía de la Mano. Madrid: Marban SL, 2007: 2049-136.

15. Lubahn JD, Wolfe TL. Joint replacement in the rheumatoid hand: surgery and therapy. En: Mackin EJ, Callahan AD, Skirven TM, et al (Eds). Rehabilitation of the hand and upper extremity. $5 t^{a}$ ed. St. Luis: Mosby, 2002: 1583-97.

16. Mandl LA, Galvin DH, Bosch JP, et al. Metacarpophalangeal arthroplasty in rheumatoid arthritis: what determines satisfaction with surgery?. J Rheumatol, 2002; 29: 2488-91. 